\title{
Functional Genomics of Cardioprotection by Ischemic Conditioning and the Influence of Comorbid Conditions: Implications in Target Identification
}

\author{
Z.V. Varga ${ }^{1, \#}$, Z. Giricz ${ }^{1, \#}$, P. Bencsik ${ }^{2,3, \#}$, R. Madonna ${ }^{4}$, M. Gyöngyösi ${ }^{5}$, R. Schulz ${ }^{6}$, M. Mayr ${ }^{7}$ \\ T. Thum ${ }^{8}$, L.G. Puskás ${ }^{9}$ and P. Ferdinandy ${ }^{1,2, *}$
}

\begin{abstract}
${ }^{I}$ Department of Pharmacology and Pharmacotherapy, Semmelweis University, Budapest, Hungary; ${ }^{2}$ Pharmahungary Group, Szeged, Hungary; ${ }^{3}$ Cardiovascular Research Group, Department of Biochemistry, University of Szeged, Szeged, Hungary; ${ }^{4}$ Center of Excellence on Aging, and Institute of Cardiology, Department of Neuroscience and Imaging, "G. d'Annunzio” University, Chieti, Italy; ${ }^{5}$ Department of Cardiology, Medical University of Vienna, Vienna, Austria; ${ }^{6}$ Department of Physiology, Justus-Liebig University, Giessen, Germany; ${ }^{7}$ King's British Heart Foundation Centre, King's College of London, London, United Kingdom; ${ }^{8}$ Institue of Molecular and Translational Therapeutic Strategies, Hannover Medical School, Hannover, Germany; ${ }^{9}$ Laboratory of Functional Genomics, Biological Research Center, Hungarian Academy of Sciences, Szeged, Hungary
\end{abstract}

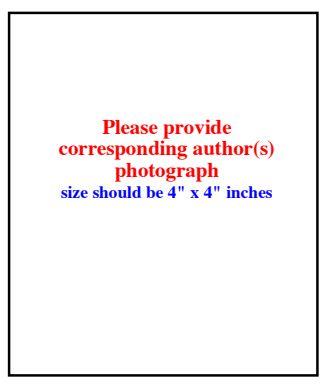

\begin{abstract}
Ischemic heart disease including myocardial infarction develops on the basis of several risk-factors and comorbidities such as obesity, diabetes, hypertension, and hypercholesterolemia. Ischemic heart disease is the leading cause of mortality worldwide, therefore, identification of novel drug targets for cardioprotection is of great importance. Ischemic preconditioning, postconditioning, and remote conditioning trigger endogenous cardioprotective mechanisms that render the heart more resistant to lethal ischemic-reperfusion injury. However, major cardiovascular co-morbidities such as hyperlipidemia, diabetes, and their co-medications interfere with these cardioprotective mechanisms thereby limiting the efficacy of cardioprotective ischemic conditioning maneuvers. Ischemia reperfusion injury and cardioprotection by conditioning have been shown to affect global myocardial gene expression profile at the transcript level. Further understanding and the comprehensive analysis of the cardioprotective gene expression fingerprint in normal, protected, and in comorbid conditions may lead to identification of novel molecular targets for cardioprotection.
\end{abstract}

Keywords: Microarray, microRNA, miRNA, mRNA, postconditioning, preconditioning, proteomics, risk factors, sequencing, system biology, transcriptomics.

\section{ISCHEMIC CONDITIONING OF THE HEART}

Ischemic conditioning leads to remarkable cardioprotection against $\mathrm{I} / \mathrm{R}$ injury (see for extensive reviews: [1, 2]). Ischemic conditioning has 3 major forms, preconditioning, postconditioning, and remote conditioning. In ischemic preconditioning, brief episodes of ischemia/reperfusion are applied before a prolonged ischemic event that would otherwise lead to significant irreversible tissue injury. Ischemic preconditioning is the most effective cardioprotective mechanism that evokes cardioprotection [3] immediately after triggering ischemia/reperfusion cycles and in a delayed manner (appearing 12-24 hours after the triggering ischemia/reperfusion cycles and lasting for approximately 72 hours) the latter termed as the second window of protection [4]. In case of ischemic postconditioning, very brief episodes of ischemia/reperfusion are applied immediately after the

*Address correspondence to this author at the Department of Pharmacology and Pharmacotherapy, Semmelweis University, Nagyvárad tér 4, Budapest, H-1089, Hungary; Tel: ?????????????; Fax: ?????????????;

E-mail: peter.ferdinandy@pharmahungary.com

\#These authors contributed equally to the present work.

$1389-4501 / 15 \$ 58.00+.00$ prolonged ischemic event. In remote ischemic conditioning, brief cycles of ischemia/reperfusion are applied to an extracardiac organ before, during, or after the prolonged ischemic event. The cardioprotective effect of pre-, post-, and remote conditioning has been well established in preclinical models as well as in clinical studies (see for reviews: [2, 5, $6]$ ), however, its effectiveness is limited by the presence of several risk factors, comorbidities, and co-medications (see for extensive reviews: $[1,7,8])$. Moreover, in spite of 3 decades of intense research on the cellular mechanism of conditioning, it is still not completely understood. Therefore, no drug targets have been identified so far that led to the development of a cardioprotective drug until market launching. This fact urges to find novel approaches to increase the translational value of preclinical studies (see for review [9]) and to find valid drug targets and novel therapeutic approaches for cardioprotection (see for review: $[1,10]$ and Table 1).

In this review, we describe how assessment of changes in cardiac gene expression profile triggered by conditioning may facilitate the development of cardioprotective drugs that mimic the remarkable cardioprotection achieved by ischemic conditioning maneuvers. 
Table 1. Examples for potential targets revealed by functional genomics approaches.

\begin{tabular}{|c|c|c|}
\hline Target & Role & Reference \\
\hline \multicolumn{3}{|l|}{ Ischemic conditioning } \\
\hline MAPKAP kinase 3 & signaling & [11] \\
\hline cathepsin G & protease & {$[11]$} \\
\hline PDE9A1 & phosphodiesterase & {$[12]$} \\
\hline peroxredoxin 4 & antioxidant defence & {$[18]$} \\
\hline PDGFR & signaling & {$[18]$} \\
\hline ZAC1 & transcription factor & {$[20]$} \\
\hline microRNA-139-5p & unknown & [17] \\
\hline microRNA-125b* & unknown & {$[17]$} \\
\hline let-7b & unknown & [17] \\
\hline microRNA-487b & unknown & [17] \\
\hline \multicolumn{3}{|l|}{ Hyperlipidemia and obesity } \\
\hline ATF3 & transcription factor & {$[25]$} \\
\hline $\begin{array}{l}\text { NADH-ubiquinone oxi- } \\
\text { doreductase }\end{array}$ & energy metabolism & {$[27]$} \\
\hline Hsp86 & stress response & {$[27]$} \\
\hline procollagen & structural proteins & [27] \\
\hline argininosuccinate synthetase & Arg metabolism & [28] \\
\hline Hsp70 & stress response & {$[28]$} \\
\hline cytochrome $\mathrm{C}$ oxidase & energy metabolism & {$[30]$} \\
\hline MMP-9 & remodeling & {$[30]$} \\
\hline microRNA-25 & oxidative stress & {$[33]$} \\
\hline microRNA-27b & hypertrophy & [34-36] \\
\hline \multicolumn{3}{|l|}{ Diabetes } \\
\hline vanilloid receptor 1 & signaling & [64] \\
\hline histone deacetylase 2 & gene regulation & [64] \\
\hline apolipoprotein B & lipid metabolism & [64] \\
\hline MMP-13 & remodeling & {$[64]$} \\
\hline microRNA-208a & hypertrophy & {$[54,55]$} \\
\hline microRNA-24 & vascularization & {$[54,55]$} \\
\hline microRNA-21 & hypertrophy & {$[54,55]$} \\
\hline
\end{tabular}

\section{GENE EXPRESSION OF THE HEART IN CARDIO- PROTECTION BY ISCHEMIC CONDITIONING}

There is a continuously increasing interest in ischemic conditioning of the heart as shown by the increasing number of papers in PubMed. Although there are a total of more than ten thousand papers in the literature on cardioprotection by ischemic conditioning, less than $1 \%$ of these studies assessed global gene expression profile of the heart in response to ischemic conditioning. These studies have shown that preconditioning and postconditioning significantly affect the gene expression profile of the ischemic heart at the transcript level. The first studies in the literature showing that early and late ischemic preconditioning affect gene expression profile of the heart using 1,2 or 3 cycles of $5 \mathrm{~min}$ ischemia/5 min perfusion in rabbits and in isolated rat hearts, respectively, are more than 10 years old [11-13]. In rabbit hearts, 35 genes with significantly altered expression patterns in response to preconditioning were discovered including upregulated MAPKAP kinase 3 and cathepsin $G$ genes in the preconditioned region, and GTP exchange factor, $\mathrm{Na}^{+} / \mathrm{K}^{+}$-ATPase, $\mathrm{Zn}$ finger protein 35 , cytochrome c oxidase, mitogen-responsive phosphoprotein, and Ran-binding protein in the nonischemic region [11]. In rat hearts, oligoadenylate synthase, chaperonin subunit epsilon, a cGMP phosphodiesterase (PDE9A1), a secretory carrier membrane protein, an amino acid transporter, and protease 28 subunit genes were changed in response to preconditioning [12].

These early studies have been confirmed by several papers showing that both ischemic preconditioning and postconditioning trigger a cardioprotective gene expression profile in the heart at the transcript level [14-17]. In line with these findings, changes in the expression of the posttranscriptional regulators of gene expression, i.e. microRNAs have been recently shown by preconditioning and postconditioning in rat hearts [18]. Whether remote ischemic conditioning may also affect global gene expression profile of the heart has not been specifically studied so far. Nevertheless, the early study of Simkhovich et al. [11] showing that the gene expression profile of the non-ischemic remote area was changed, suggests that remote conditioning also leads to a cardioprotective transcriptomic program. Accordingly, one paper has reported cardiac gene expression profile alterations following remote ischemic preconditioning; e.g. upregulation of genes involved in oxidative stress (e.g. peroxiredoxin 4, platelet-derived growth factor receptor) 15 min or 24 hours after 6 cycles of 4-min femoral artery occlusion/reperfusion in mice [19]. In line with this assumption, extracellular vesicles, potential carriers of microRNAs, have been shown to mediate the cardioprotective effect of remote ischemic preconditioning induced by $3 \times 5-5 \mathrm{~min}$ ische$\mathrm{mia} /$ reperfusion cycles in rat hearts [20].

Identification of global gene expression profile of the heart in response to ischemic conditioning may help to identify key cellular pathways of ischemia/reperfusion injury and cardioprotection by ischemic conditioning. The transcription factor ZAC1 gene has been identified as potential target for gene silencing as this gene was significantly down-regulated by preconditioning and postconditioning [21]. Similarly, by a systematic comparison method looking at the direction of microRNA expression changes after ischemia/reperfusion injury with or without preceding preconditioning or subsequent postconditioning, respectively, potential cardioprotective microRNA targets have been identified and termed "protectomiRs", e.g. mimics of microRNA-139-5p, -125b*, let$7 \mathrm{~b}$, and antagomiR of microRNA-487b in rat hearts [18]. 
These preclinical results show that by assessing the cardioprotective gene expression profile of the heart subjected to ischemia/reperfusion with or without cardioprotection elicited by ischemic conditioning, novel targets for cardioprotection can be identified. However, no data on the cardioprotective gene expression fingerprints in the human heart are available so far.

\section{INFLUENCE OF CO-MORBIDITIES ON CARDIAC GENE EXPRESSION PATTERN}

The mechanism by which the remarkable cardioprotective effect of ischemic conditioning is attenuated or abolished in the presence of major cardiovascular risk factors and comorbidities as well as their routine drug treatments is not exactly known (see for extensive reviews: $[1,7,8]$ ). Therefore, for successful cardioprotective target identification and validation, it is of great importance to assess the effect of major cardiovascular comorbidities and co-medications such as dyslipidemia, diabetes, hypertension, obesity, aging etc (see for review: [1]). Accentuated myocardial oxidative stress has been reported in the presence of major comorbidities. Therefore, it is plausible to speculate that redox signaling-dependent global gene expression changes contribute to the pathological phenotypes. The redox-sensitive modulation of gene expression may occur both by direct oxidative/nitrative modification of the transcription factor itself or by posttranslational modifications (i.e. mainly phosphorylation/dephosphorylation] due to alterations in redox-regulated intracellular signaling cascades (e.g. p38 MAPK) [22]. So far limited amount of data have been accumulated in the literature regarding the effect of hyperlipidemia, obesity, and diabetes on cardiac gene expression profile, while there are still negligible data on other cardiovascular risk factors and comorbidities. In this chapter we will review the current data available in the literature on the effect of major cardiovascular comorbidities on cardiac gene expression profile, focusing on metabolic diseases such as hyperlipidemia, obesity and diabetes.

\subsection{Hyperlipidemia, Obesity and Statins}

Hyperlipidemia and obesity are amongst the most important risk factors for cardiovascular diseases due to their steeply increasing prevalence worldwide. Moreover, hyperlipidemia was the first risk factor to be associated with the loss of cardioprotection by ischemic preconditioning in rabbits and rats on chronic cholesterol-enriched diet ([23, 24], see for the first extensive review [8]). Since then, it has been well established that most of the other major risk factors and their medications may modify cardioprotective signaling $[1,7]$. Therefore, to better characterize the effect of these pathologies on the heart and to uncover novel therapeutic targets, several research groups have performed systematic analyses of cardiac gene expression in various disease models.

A few publications aimed to decipher how the composition of consumed excess fat influences gene expression in the heart. Lockridge and colleagues hypothesized that exposure to various fatty acids alone have differential effect on the gene expression pattern of isolated cardiomyocytes. They compared effects of fatty acids considered cardioprotective and cardiotoxic by cDNA microarrays and revealed that $24 \mathrm{~h}$ treatment of saturated fatty acids tended to induce endoplasmic reticulum- and oxidative stress markers (e.g. activating transcription factor 3 or growth arrest and DNA-damageinducible protein $\alpha$ ) more than a treatment with unsaturated fatty acids [25]. The acute effects of unsaturated fatty acids on gene expression in the heart were studied in-vivo as well. Mice were fed with triglycerides comprising specific fatty acids, and 6 hour later mRNA expression profiles of the hearts were determined. Similarly to the experiment on isolated cells, a differential modulation of gene expression was shown, where linolenic acid (C18:3) modulated the expression of most genes, the majority of which was related to the PPAR $\alpha$ signaling pathway (e.g. heme oxygenase 1 or angiopoietin-like 4 protein) [26]. These studies clearly demonstrate that even a short term overload of dietary fats might modulate cardiac gene expression, and that these effects are specific to fatty acid moieties and most likely not a direct consequence of the cellular energy imbalance.

Long term effects of hyperlipidemia and obesity on cardiac gene expression has been studied in several papers. In an early study, Puskas et al reported that in the hearts of rats on cholesterol-enriched chow for two months expression of numerous genes were modulated, including members of energy metabolism (e.g. NADH-ubiquinone oxidoreductase), heat shock proteins (heat shock protein 86), ion channels (sodium/potassium ATPase), and structural proteins (procollagen, type III, $\alpha 1$ ) [27]. Later, Sarkozy et al described differences in gene expression between Zucker diabetic fatty and control lean rats including several metabolic enzymes (e.g. argininosuccinate synthetase), stress response proteins (e.g. heat shock protein 70,1A) and ion channels (e.g. sodium/potassium ATPase $\beta 4$ polypeptide) [28]. Several of these genes have not been implicated in cardiovascular diseases before. Tissue specific gene expression changes were also compared in obese animals [29]. In high-fat chow fed mice expression of several hundred genes is altered, although only a fraction of them is common between tissues. In the heart most robust changes were detected in members of lipid metabolism (e.g. major urinary proteins 2,3 and 5), but in contrast, in the adipose tissue expression of several genes of the immune system were altered (e.g. CD84 antigen). In addition, a profound modulation of PPAR signaling in most tissues is shown in this publication, similarly to others, e.g. [26].

Large animal models have been rarely used for cardiac transcriptomics studies. Rouet and colleagues investigated dogs after 9 weeks of high fat diet that lead to approximately $25 \%$ weigh gain and arterial hypertension. This treatment resulted in a profound alteration of atrial and ventricular gene expression [30]. They identified several metabolic enzymes (e.g. cytochrome C oxidase), ion channels (e.g. sodium/potassium ATPase), genes involved in protein synthesis and remodeling (e.g. matrix metalloprotease 9 , or nuclear factor erythroid 2-related factor 2). Interestingly, most gene expression changes have been detected in the atria and not the left ventricle. For example, they assessed a decrease in phospholamban and sarco-endoplasmic reticulum $\mathrm{Ca}^{2+}$ ATPase-2 (SERCA-2) mRNA level only in the atria. In a following study, they studied temporal changes in gene expression as well. Dogs were fed with high-fat chow for 9-24 weeks and left ventricular mRNA levels were assessed by 
microarrays [31]. In these experiments they identified a timedependent decrease in the ventricular expression of phospholamban and SERCA-2. At 24 weeks they also found a decrease in myostatin expression, and an up-regulation in the expression of several genes related to the TGF- $\beta$ pathway, which are also implicated in obesity-related cardiac pathologies, such as hypertrophy and excessive fibrosis.

The effect of human obesity or hyperlipidemia on cardiac gene expression patterns is not well studied. Philip-Couderc et al investigated mRNA transcript levels from right atrial appendages of obese and lean patients. They reported that obesity modulates the expression of almost 400 genes in the heart, and showed that the most affected signaling pathway was the down-regulated Wnt system, which is involved in cardiac hypertrophy [32]. Although this report shows that the gene expression pattern of the human atrium is severely altered in obesity, so far data is unavailable from ventricular samples. Given that the overlap in reported gene expression changes between species and models seem to be low, studies on obesity- and hyperlipidemia-modulated gene expression is still warranted.

During the last decade an increasing number of researchers directed their focus on the systematic analysis of miRNAs in the cardiovascular system. We recently reported that expression of several miRNAs is altered in the ventricles of rats fed a cholesterol-enriched chow [33]. One of the most robust down-regulation was found in miR-25, which might be responsible for the increased oxidative- and nitrosative stress observed in this model, since one of the putative targets of miR-25 is NOX4, a major source of reactive oxygen species. The role of miRNAs in hyperlipidemia- and obesityinduced changes in gene expression was also studied in the liver of mice on high-fat diet [34]. They found that hepatic expression of miR-27b is elevated in obese animals. The study concluded that miR-27b is a key regulator of hepatic lipid metabolism since the expression of its several target genes were also altered in hyperlipidemia. One might suggest that cardiac lipid metabolism can also be influenced by miRNAs through the circulation. Indeed, it has been reported that the overexpression of miR-27b leads to cardiac dysfunction and hypertrophy in mice [35], however, elsewhere it was identified as an anti-hypertrophic miRNA [36]. As in case of mRNA transcriptomics studies, data on cardiac expression of miRNAs in hyperlipidemic or obese humans is missing.

Statins, as inhibitors of 3-hydroxy-3-methyl glutaryl coenzyme A reductase, are potent cholesterol-lowering drugs that are widely used in clinical practice for primary and secondary prevention of coronary heart disease. Statins can exert pleiotropic effects aside from lowering cholesterol and blood pressure through several different pathways including the induction of endothelial nitric-oxide synthase expression, anti-inflammatory actions, and antioxidant activity [37]. We have previously shown in isolated rat hearts that lovastatin interferes with the anti-ischemic effect of ischemic pre- and postconditioning [38]. However, only a handful of preclinical studies investigated the effects of statin treatment on cardiac gene expression pattern. In a comparative study different types of statins such as atorva-, prava-, pitava-, and rosuvastatin were administered orally to normal $\mathrm{C} 57 \mathrm{Bl} / 6$ mice for 4 weeks [39]. The authors found altogether 49 genes to be up- or down-regulated by statin treatments in a drugspecific manner, from which tissue inhibitor of metalloproteinases-3 showed the most robust increase in response to atorvastatin treatment. Others reported on cardiac expression changes of 93 genes after 4-week atorvastatin [15 $\mathrm{mg} / \mathrm{kg}$ /day) treatment in normal Wistar Kyoto rats, whilst cardiac mRNA level of 60 genes has been altered by the same treatment in stroke prone spontaneously hypertensive rats [40]. The altered genes included several ones involved in general metabolic pathways (glucose, fatty acid, and cholesterol biosynthesis, etc.), cell division, signaling, motility, cell/organism defense, regulation of protein expression, oxidative stress, and inflammatory processes. Despite the abundance of clinical studies investigating the effects of statins, comprehensive studies on the effect of statins on cardiac gene expression profiles in the healthy and ischemic human myocardium are still missing.

\subsection{Diabetes}

Diabetes-related metabolic and molecular alterations ultimately lead to complex and serious cardiovascular complications, involving accelerated progression of atherosclerosis and deterioration of cardiac function [41). Diabetic myocardial dysfunction and diabetic cardiomyopathy is the factor that is responsible for higher cardiac morbidity and mortality in diabetics [42]. It is well established now that multiple factors contribute and promote to the development and evolution of diabetic cardiomyopathy (these include hyperglycemia and subsequent insulin resistance, increased fatty acid metabolism, microcirculatory changes, sympathetic dysfunction, myocardial inflammation, oxidative/nitrative stress, remodeling and fibrosis), however the exact molecular events that initiate and fuel these pivotal pathological mechanisms are not entirely clear [43]. To understand the underlying biological processes and identify new molecular targets, detailed characterization of gene expression in these hearts with systems biological tools is of high importance.

Investigations in pre-diabetic models are clinically essential, due to the enormously increasing incidence and overlapping prevalence of metabolic syndrome and pre-diabetes [44]. There is also evidence for the deterioration of myocardial function in models of pre-diabetes [45-47], in line with these, recently we have reported marked global gene expression alterations in the heart Zucker diabetic fatty rats [28], a model that is characterized by main features of pre-diabetes and metabolic syndrome (obesity, hyperglycemia, hyperinsulinemia, and hypercholesterolemia). Among the 14921 transcript assessed, 10244 showed detectable expression in the heart. Altogether 85 transcripts showed significant alterations (up- or down-regulation), when compared to the lean control rats. The detailed analysis (involving functional clusterization and gene ontology analysis) showed alterations in transcripts of lipid metabolism (e.g. 3-hydroxy-3methylglutaryl-Coenzyme A synthase 2; argininosuccinate synthetase; 2-amino-3-ketobutyrate-coenzyme A ligase), extra- and intracellular structural proteins (e.g. myosin IXA; aggrecan1), signal transduction (e.g. activating transcription factor 3; phospholipase A2) stress response (e.g. heat shock $70 \mathrm{kD}$ protein $1 \mathrm{~A}$; heat shock protein 60 ; glutathione $\mathrm{S}$ transferase $\mathrm{Yc} 2$ subunit), ion channels and receptors (e.g. ATPase, $\mathrm{Na}^{+} / \mathrm{K}^{+}$transporting, beta 4 polypeptide; ATPase, 
$\mathrm{H}^{+} / \mathrm{K}^{+}$transporting, non-gastric, alpha polypeptide). These profound modifications in the cardiac transcriptome might be involved in the development of cardiac pathological abnormalities induced by pre-diabetes and metabolic syndrome.

Similar comprehensive studies were carried out in recent years in different models of overt diabetes, either using genetic models of the disease (Goto-Kakizaki diabetic rats, Otsuka Long-Evans Tokushima fatty rats) or the streptozotocin-induced type I. diabetes model. Karakikes et al. revealed significant divergence in myocardial gene expression profile in the hearts of the diabetic Otsuka Long-Evans Tokushima fatty rats when compared to the non-diabetic controls [48]. Out of the 20500 transcripts surveyed, 838 showed divergent expression (272 up-regulations and 566 down-regulations). Their subsequent functional analysis indicates that diabetic cardiomyopathy in this type 2 diabetes model is associated with changes in transcripts involved in immunity, development, intracellular signaling, cell proliferation and transcription regulation.

There is also evidence for the profound effect of diabetes on deteriorated post-infarction remodeling. The adverse remodeling process in diabetes is in association with profound myocardial gene expression alterations. The study by Song et al. suggest that in type 1 diabetic rats transcriptomic changes are responsible for altered myocardial metabolic substrate utilization (affected fatty acid metabolism, carboxylic acid synthesis, steroid metabolisms), that may account for the exaggerated progression of post-infarction remodeling processes [49].

Gestational diabetes is a specific diabetic condition, affecting both the health of the-mother and the baby. Although, there is a three- to four fold increase in the incidence of congenital heart defects caused by maternal diabetes [50], the exact molecular mechanisms that contribute to the teratogenic effects of the diabetic milieu are not known. A recent study investigated gene expression profiles of developing hearts of embryos, aiming to shed light on the mechanisms of cardiac malformation development [51]. The study revealed several differentially expressed genes in healthy and diabetic embryonic hearts. Based on the functional categorization of the altered transcripts it is hypothesized that genes involved in lipid metabolism, cell cycle and transcription regulation have important role in cardiac embryopathy.

Although, transcriptomic tools have revealed several important gene expression alterations in the heart in diabetic conditions, several limitations have to be noted. In the last years it became evident that the fate of the mRNA is determined by presence of other regulatory non-coding RNA species (e.g. microRNAs). In addition the importance and extent of alternative splicing events in cardiac pathologies are now recognized [52] that are impossible to assess with conventional microarray-based tools. In line with these, reactivation of the fetal splicing gene program has been described by Verma et al. [53]. RNA sequencing and subsequent bioinformatics identification of splicing events revealed that several important transcripts implicated in diabetes (e.g. VEGF, Mef2a) undergo alternative splicing. In addition there is a significant similarity of splicing pattern in the diabetic and developing embryonic heart, indicating the reprogramming of fetal gene expression events.
Recent studies also indicate the importance of microRNAs in the development of diabetic myocardial pathologies. The concordant studies of the Hu lab indicate striking alterations in microRNA expression profile in the heart of streptozotocin treated rats, implicating alterations in whole gene networks [54, 55]. They identified 19 microRNAs that were dysregulated in diabetic hearts (up-regulation of miR-195, miR-199a-3p, miR-142-3p, miR-24, miR-21, miR-208a, miR-221, miR-499-3p, miR-700, and miR-705 and downregulation of miR-1, miR-143, miR-29, miR-20a, miR-220b, and miR-373). Based on bioinformatic target prediction and partial experimental validation, it seems that the affected microRNAs have a significant fingerprint on myocardial transcriptomic profile (affected genes involve hypertrophyand fibrosis-related targets).

In addition, accumulating evidence proves the applicability of microRNAs as diagnostic biomarkers. In a landmark study Zampetaki et al. determined plasma microRNA expression profiles in diabetic patients [56], revealing 5 candidate microRNAs (miR-15a, miR-29b, miR-126, miR-223, and miR-28-3p) to be used as diagnostic indicators and predictors of later onset diabetes. Another study recently addressed altered circulating miRNA levels in children with type I diabetes; here interestingly levels of miR-126 were predominantly down in urine but not plasma of patients suggesting more pathological involvement of the kidney in response to type I diabetes [57).

The prevalence of diabetic neuropathy is greater than $50 \%$ in patients with long-standing diabetes [58]. Although, several papers have been published investigating the gene expression changes in different neural structures during the development of diabetic neuropathy, it is not clear if diabetes affects cardiac gene expression due to sensory and/or motor neuropathy. Capsaicin, an essential compound of hot pepper, is used as topical treatment to alleviate severe pain in patients with diabetic neuropathy [59]. Capsaicin is a highly selective sensory neurotoxin that leads to a selective functional blockade of a primary sensory neurons $[60,61]$. We have previously shown that capsaicin-sensitive sensory nerves play an important role in preconditioning in rat hearts [62]. Furthermore, we demonstrated that capsaicin-sensitive nerves are involved in the regulation cardiac NO-cGMP signaling [63], which is known to be one of the major regulatory pathways for ischemic conditioning. Moreover, we have also shown that selective chemodenervation of capsaicinsensitive sensory nerves in male Wistar rats in vivo, beyond cardiac diastolic dysfunction, induced changes in myocardial gene expression [64]. In this study, among the 6400 rat genes examined by DNA microarray and/or by QRT-PCR, 47 genes exhibited significant up-regulation and 36 were downregulated. The up-regulated genes affect neural functions (e.g. vanilloid receptor 1; GABA receptor rho-3 subunit), signal transduction (including NO signaling), cell adhesion (integrin $\alpha \mathrm{v}$ subunit), gene regulation (histone deacetylase 2 ), and metabolic pathways particularly the lipid (apolipoprotein B, farnesyl transferase) metabolism. Among the repressed genes we have also found genes of metabolic pathways (e.g. aldehyde dehydrogenase), cell cycle regulators (cyclin-dependent kinase 4), matrix metalloproteinase 13, and interleukin 7 . 
A

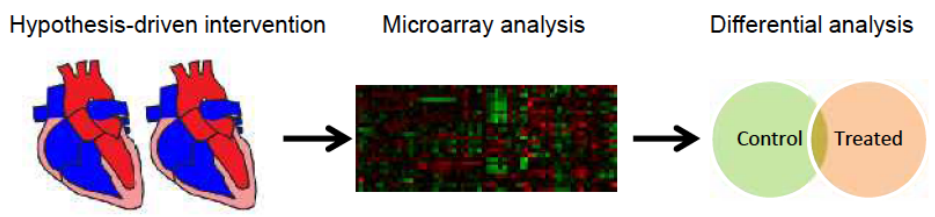

B

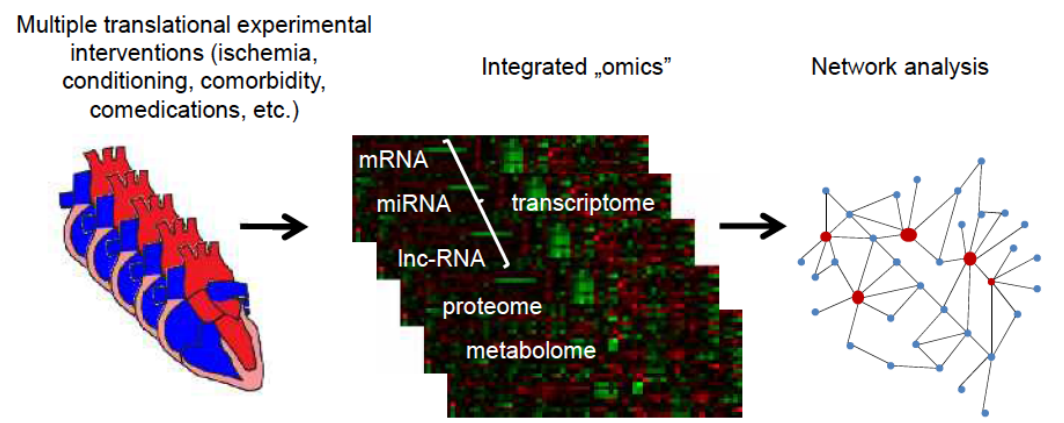

Fig. (1). Approaches of functional genomics. Panel A shows a conventional hypothesis-driven approach to investigate differentially expressed transcripts induced by a single experimental intervention. A more comprehensive way is to compare multiple expression patterns on different levels of functional genomics (mRNA, microRNA, long non-coding RNA) and proteomics and to integrate these "omics" data by network analysis to identify novel signalling hubs and networks. The array images were created by matrix 2 png algorithm [68].

\section{CONCLUSION AND PERSPECTIVE}

By assessing global cardiac gene expression profile of the ischemic heart with or without cardioprotection by ischemic conditioning, one may explore the cardioprotective gene expression fingerprint and identify novel drug targets for cardioprotection (Table 1). However, for identification of valid drug targets, the effect of cardiovascular risk factors, comorbidities, and co-medications of ischemic heart disease should be taken into account. There is also an unmet need for studies that utilize an integrated "omics" approach (mRNA and non-coding RNA transcriptomics followed by proteomic analysis) with subsequent robust bioinformatics (e.g. network analysis) to identify key regulatory networks and signaling hubs in response to cardioprotection (Fig. (1) [65, 66]). This approach may provide the basis of cardioprotective gene threrapy as well [67]. So far very few studies looked at changes in the gene expression profile of the ischemic heart in response to cardioprotection and its confounding factors. However, such studies would be necessary to identify key pathways of cardioprotection that are still active in the presence of major cardiovascular comorbidities and their routine medications.

\section{LIST OF ABBREVIATIONS}

$\begin{array}{lll}\mathrm{I} / \mathrm{R} & = & \text { Ischemia/reperfusion } \\ \text { MAPKAP kinase } & = & \text { Mitogen-activated protein kinase- } \\ & \text { activated protein kinase } \\ \text { Mef2 } & = & \text { Myocyte enhancer factor-2 } \\ \text { NO-cGMP } & = & \text { Nitric oxide, cyclic GMP } \\ \text { NOX4 } & = & \text { NADPH oxidase } 4 \\ \text { PPAR } \alpha & = & \text { Peroxisome proliferator-activated } \\ & \text { receptor alpha }\end{array}$

$$
\begin{array}{lll}
\text { QRT-PCR } & = & \text { Quantitative real time polymerase } \\
\text { SERCA-2 } & = & \text { Sarco-endoplasmic reticulum } \mathrm{Ca}^{2+}- \\
& \text { ATPase-2 }
\end{array}
$$

\section{CONFLICT OF INTEREST}

The authors confirm that this article content has no conflict of interest.

\section{ACKNOWLEDGEMENTS}

This work was supported by the following grants: Hungarian Scientific Research Fund and the Austrian Science Fund (OTKA ANN 107803). ZG and PB hold „János Bolyai Fellowship" from the Hungarian Academy of Sciences. ZVV was supported by the National Program of Excellence (TAMOP 4.2.4.A/1-11-1-2012-0001and by the Scientific Merit Award of the Semmelweis University. PF was a Szentágothai Fellow of the National Program of Excellence (TAMOP 4.2.4.A/2-11-1-2012-0001).

\section{REFERENCES}

[1] Ferdinandy P, Hausenloy DJ, Heusch G, Baxter GF, Schulz R Interaction of risk factors, comorbidities, and comedications with ischemia/reperfusion injury and cardioprotection by preconditioning, postconditioning, and remote conditioning. Pharmacol Rev 2014; 66: 1142-74.

[2] Hausenloy DJ, Erik Botker H, Condorelli G, et al. Translating cardioprotection for patient benefit: position paper from the Working Group of Cellular Biology of the Heart of the European Society of Cardiology. Cardiovasc Res 2013; 98: 7-27.

[3] Lawson CS, Downey JM. Preconditioning: state of the art myocardial protection. Cardiovasc Res 1993; 27: 542-50.

[4] Bolli R. The late phase of preconditioning. Circulation Research 2000; 87: 972-83.

[5] Ovize M, Baxter GF, Di Lisa F, et al. Postconditioning and protection from reperfusion injury: where do we stand? Position 
paper from the Working Group of Cellular Biology of the Heart of the European Society of Cardiology. Cardiovasc Res 2010; 87: 406-23.

[6] Baxter GF, Ferdinandy P. Delayed preconditioning of myocardium: current perspectives. Basic Res Cardiol 2001; 96: 329-44.

Ferdinandy P, Schulz R, Baxter GF. Interaction of cardiovascular risk factors with myocardial ischemia/reperfusion injury, preconditioning, and postconditioning. Pharmacol Rev 2007; 59: 418-58.

[8] Ferdinandy P, Szilvassy Z, Baxter GF. Adaptation to myocardial stress in disease states: is preconditioning a healthy heart phenomenon? Trends Pharmacol Sci 1998; 19: 223-9.

[9] Lecour S, Botker HE, Condorelli G, et al. ESC Working Group Cellular Biology of the Heart: Position Paper: improving the preclinical assessment of novel cardioprotective therapies. Cardiovasc Res 2014; 104: 399-411.

[10] Sluijter JP, Condorelli G, Davidson SM, et al. Novel therapeutic strategies for cardioprotection. Pharmacol Ther 2014; 144: 60-70.

[11] Simkhovich BZ, Abdishoo S, Poizat C, Hale SL, Kedes LH, Kloner RA. Gene activity changes in ischemically preconditioned rabbit heart gene: discovery array study. Heart Disease 2002; 4: 63-9.

[12] Onody A, Zvara A, Hackler L, Jr., Vigh L, Ferdinandy P, Puskas LG. Effect of classic preconditioning on the gene expression pattern of rat hearts: a DNA microarray study. FEBS Letters 2003; 536: 35-40.

[13] Zubakov D, Hoheisel JD, Kluxen FW, et al. Late ischemic preconditioning of the myocardium alters the expression of genes involved in inflammatory response. FEBS Letters 2003; 547: 51-5.

[14] Kocsis GF, Csont T, Varga-Orvos Z, Puskas LG, Murlasits Z, Ferdinandy P. Expression of genes related to oxidative/nitrosative stress in mouse hearts: effect of preconditioning and cholesterol diet. Med Sci Monit 2010; 16: BR32-9.

[15] Csonka C, Szucs G, Varga-Orvos Z, et al. Ischemic postconditioning alters the gene expression pattern of the ischemic heart. Exp Biol Med (Maywood) 2014; 239: 141-50.

[16] Simkhovich BZ, Marjoram P, Poizat C, Kedes L, Kloner RA. Brief episode of ischemia activates protective genetic program in rat heart: a gene chip study. Cardiovasc Res 2003; 59: 450-9.

[17] Faragó N, Kocsis GF, Fehér LZ, et al. Gene and protein expression changes in response to normoxic perfusion in mouse hearts. $\mathrm{J}$ Pharmacol Toxicol Methods 2008; 57: 145-54.

[18] Varga ZV, Zvara A, Farago N, et al. MicroRNAs associated with ischemia-reperfusion injury and cardioprotection by ischemic preand postconditioning: protectomiRs. Am J Physiol Heart Circ Physiol 2014; 307: H216-27.

[19] Garibaldi BT, D'Alessio FR, Mock JR, et al. Regulatory T cells reduce acute lung injury fibroproliferation by decreasing fibrocyte recruitment. Am J Respir Cell Mol Biol 2013; 48: 35-43.

[20] Giricz Z, Varga ZV, Baranyai T, et al. Cardioprotection by remote ischemic preconditioning of the rat heart is mediated by extracellular vesicles. J Mol Cell Cardiol 2014; 68: 75-8.

[21] Vincent A, Gahide G, Sportouch-Dukhan C, et al. Down-regulation of the transcription factor ZAC1 upon pre- and postconditioning protects against I/R injury in the mouse myocardium. Cardiovasc Res 2012; 94: 351-8.

[22] D'Alterio C, Barbieri A, Portella L, et al. Inhibition of stromal CXCR4 impairs development of lung metastases. Cancer Immunol Immunother 2012; 61: 1713-20.

[23] Szilvassy Z, Ferdinandy P, Szilvassy J, et al. The loss of pacinginduced preconditioning in atherosclerotic rabbits: role of hypercholesterolaemia. J Mol Cell Cardiol 1995; 27: 2559-69.

[24] Ferdinandy P, Szilvássy Z, Horváth LI, et al. Loss of pacinginduced preconditioning in rat hearts: role of nitric oxide and cholesterol-enriched diet. Journal of Molecular and Cellular Cardiology. 1997; 29: 3321-33.

[25] Lockridge JB, Sailors ML, Durgan DJ, et al. Bioinformatic profiling of the transcriptional response of adult rat cardiomyocytes to distinct fatty acids. J Lipid Res 2008; 49: 1395-408.

[26] Georgiadi A, Boekschoten MV, Muller M, Kersten S. Detailed transcriptomics analysis of the effect of dietary fatty acids on gene expression in the heart. Physiol Genomics 2012; 44: 352-61.

[27] Puskas LG, Nagy ZB, Giricz Z, et al. Cholesterol diet-induced hyperlipidemia influences gene expression pattern of rat hearts: a DNA microarray study. FEBS Letters 2004; 562: 99-104.
[28] Sarkozy M, Zvara A, Gyemant N, et al. Metabolic syndrome influences cardiac gene expression pattern at the transcript level in male ZDF rats. Cardiovasc Diabetol 2013; 12: 16

[29] Lee RK, Hittel DS, Nyamandi VZ, et al. Unconventional microarray design reveals the response to obesity is largely tissue specific: analysis of common and divergent responses to dietinduced obesity in insulin-sensitive tissues. Appl Physiol Nutr Metab 2012; 37: 257-68.

[30] Philip-Couderc P, Smih F, Pelat M, et al. Cardiac transcriptome analysis in obesity-related hypertension. Hypertension 2003; 41: 414-21.

[31] Philip-Couderc P, Smih F, Hall JE, et al. Kinetic analysis of cardiac transcriptome regulation during chronic high-fat diet in dogs. Physiol Genomics 2004; 19: 32-40.

[32] Philip-Couderc P, Pathak A, Smih F, et al. Uncomplicated human obesity is associated with a specific cardiac transcriptome: involvement of the Wnt pathway. FASEB J 2004; 18: 1539-40.

[33] Varga ZV, Kupai K, Szucs G, et al. MicroRNA-25-dependent upregulation of NADPH oxidase 4 (NOX4) mediates hypercholesterolemia-induced oxidative/nitrative stress and subsequent dysfunction in the heart. J Mol Cell Cardiol 2013; 62: 111-21.

[34] Vickers KC, Shoucri BM, Levin MG, et al. MicroRNA-27b is a regulatory hub in lipid metabolism and is altered in dyslipidemia. Hepatology 2013; 57: 533-42.

[35] Wang J, Song Y, Zhang Y, et al. Cardiomyocyte overexpression of miR-27b induces cardiac hypertrophy and dysfunction in mice. Cell Res 2012; 22: 516-27.

[36] Jentzsch C, Leierseder S, Loyer X, et al. A phenotypic screen to identify hypertrophy-modulating microRNAs in primary cardiomyocytes. J Mol Cell Cardiol 2012; 52: 13-20.

[37] Werner N, Nickenig G, Laufs U. Pleiotropic effects of HMG-CoA reductase inhibitors. Basic Research in Cardiology 2002; 97: 10516.

[38] Kocsis GF, Pipis J, Fekete V, et al. Lovastatin interferes with the infarct size-limiting effect of ischemic preconditioning and postconditioning in rat hearts. Am J Physiol Heart Circ Physiol 2008; 294: H2406-9.

[39] Kumazaki M, Ando H, Ushijima K, Fujimura A. Comparative effects of statins on murine cardiac gene expression profiles in normal mice. Eur J Pharmacol 2013; 707: 71-7.

[40] Kato N, Liang YQ, Ochiai Y, Jesmin S. Systemic evaluation of gene expression changes in major target organs induced by atorvastatin. Eur J Pharmacol 2008; 584: 376-89.

[41] Authors/Task Force M, Ryden L, Grant PJ, et al. ESC Guidelines on diabetes, pre-diabetes, and cardiovascular diseases developed in collaboration with the EASD: the Task Force on diabetes, prediabetes, and cardiovascular diseases of the European Society of Cardiology (ESC) and developed in collaboration with the European Association for the Study of Diabetes (EASD). Eur Heart J 2013; 34: 3035-87.

[42] Aksnes TA, Kjeldsen SE, Rostrup M, Omvik P, Hua TA, Julius S Impact of new-onset diabetes mellitus on cardiac outcomes in the Valsartan Antihypertensive Long-term Use Evaluation (VALUE) trial population. Hypertension 2007; 50: 467-73.

[43] Varga ZV, Giricz Z, Liaudet L, Hasko G, Ferdinandy P, Pacher P. Interplay of oxidative, nitrosative/nitrative stress, inflammation, cell death and autophagy in diabetic cardiomyopathy. Biochim Biophys Acta 44. Grundy SM. Pre-diabetes, metabolic syndrome, and cardiovascular risk. Journal of the American College of Cardiology 2012; 59: 635-43.

[44] Montaigne D, Marechal X, Coisne A, et al. Myocardial contractile dysfunction is associated with impaired mitochondrial function and dynamics in type 2 diabetic but not in obese patients. Circulation 2014; 130: 554-64.

[45] Horgan S, Watson C, Glezeva N, Baugh J. Murine models of diastolic dysfunction and heart failure with preserved ejection fraction. J Card Fail 2014; 20: 984-95.

[46] Radovits T, Korkmaz S, Loganathan S, et al. Comparative investigation of the left ventricular pressure-volume relationship in rat models of type 1 and type 2 diabetes mellitus. American Journal of Physiology Heart and Circulatory Physiology 2009; 297: H12533.

[47] Karakikes I, Kim M, Hadri L, et al. Gene remodeling in type 2 diabetic cardiomyopathy and its phenotypic rescue with SERCA2a. PloS One 2009; 4: e6474. 
[48] Song GY, Wu YJ, Yang YJ, et al. The accelerated post-infarction progression of cardiac remodelling is associated with genetic changes in an untreated streptozotocin-induced diabetic rat model. Eur J Heart Fail 2009; 11: 911-21.

[49] Wren C, Birrell G, Hawthorne G. Cardiovascular malformations in infants of diabetic mothers. Heart 2003; 89: 1217-20.

[50] Vijaya M, Manikandan J, Parakalan R, Dheen ST, Kumar SD, Tay SS. Differential gene expression profiles during embryonic heart development in diabetic mice pregnancy. Gene 2013; 516: 218-27.

[51] Lara-Pezzi E, Gomez-Salinero J, Gatto A, Garcia-Pavia P. The alternative heart: impact of alternative splicing in heart disease. $\mathrm{J}$ Cardiovasc Transl Res 2013; 6: 945-55.

[52] Verma SK, Deshmukh V, Liu P, et al. Reactivation of fetal splicing programs in diabetic hearts is mediated by protein kinase $\mathrm{C}$ signaling. J Biol Chem 2013; 288: 35372-86.

[53] Diao X, Shen E, Wang X, Hu B. Differentially expressed microRNAs and their target genes in the hearts of streptozotocininduced diabetic mice. Mol Med Rep 2011; 4: 633-40.

[54] Shen E, Diao X, Wang X, Chen R, Hu B. MicroRNAs involved in the mitogen-activated protein kinase cascades pathway during glucose-induced cardiomyocyte hypertrophy. Am J Pathol 2011; 179: 639-50.

[55] Zampetaki A, Kiechl S, Drozdov I, et al. Plasma microRNA profiling reveals loss of endothelial miR-126 and other microRNAs in type 2 diabetes. Circulation Res 2010; 107: 810-7.

[56] Osipova J, Fischer DC, Dangwal S, et al. Diabetes-associated microRNAs in pediatric patients with type 1 diabetes mellitus: a cross-sectional cohort study. J Clin Endocrinol Metabol 2014; 99 : E1661-5.

[57] Callaghan BC, Cheng HT, Stables CL, Smith AL, Feldman EL. Diabetic neuropathy: clinical manifestations and current treatments. Lancet Neurol 2012; 11: 521-34.
[58] Zilliox L, Russell JW. Treatment of diabetic sensory polyneuropathy. Curr Treat Options Neurol 2011; 13: 143-59.

[59] Jancso G, Kiraly E, Jancso-Gabor A. Pharmacologically induced selective degeneration of chemosensitive primary sensory neurones. Nature 1977; 270: 741-3.

[60] Holzer P. Capsaicin: cellular targets, mechanisms of action, and selectivity for thin sensory neurons. Pharmacol Rev 1991; 43: 143201.

[61] Ferdinandy P, Csont T, Csonka C, et al. Capsaicin-sensitive local sensory innervation is involved in pacing-induced preconditioning in rat hearts: role of nitric oxide and CGRP? NaunynSchmiedeberg's Archives of Pharmacology 1997; 356: 356-63.

[62] Csont T, Csonka C, Kovacs P, Jancso G, Ferdinandy P. Capsaicinsensitive sensory neurons regulate myocardial nitric oxide and cGMP signaling. European J Pharmacol 2003; 476: 107-13.

[63] Zvara A, Bencsik P, Fodor G, et al. Capsaicin-sensitive sensory neurons regulate myocardial function and gene expression pattern of rat hearts: a DNA microarray study. FASEB J 2006; 20: 160-2

[64] Marziliano N, Grasso M, Pilotto A, et al. Transcriptomic and proteomic analysis in the cardiovascular setting: unravelling the disease? Journal of Cardiovascular Medicine 2009; 10: 433-42.

[65] Langley SR, Dwyer J, Drozdov I, Yin X, Mayr M. Proteomics: from single molecules to biological pathways. Cardiovasc Res 2013; 97: 612-22.

[66] Madonna R, Cadeddu C, Deidda M, et al. Cardioprotection by gene therapy: A review paper on behalf of the Working Group on Drug Cardiotoxicity and Cardioprotection of the Italian Society of Cardiology. Int J Cardiol 2015; 191: 203-210.

[67] Pavlidis P, Noble WS. Matrix2png: a utility for visualizing matrix data. Bioinformatics 2003; 19: 295-6. 